\title{
Exposure to PbSe Nanoparticles and Male Reproductive Damages in a Rat Model
}

Qixing Zhou ${ }^{\dagger} *$, Zongkai Yue ${ }^{\dagger}$, Qingzhao $\mathrm{Li}^{\ddagger}$, Ruiren $\mathrm{Zhou}^{\S}$, $\mathrm{Lu} \mathrm{Liu}^{\dagger}$

${ }^{\dagger}$ Key Laboratory of Pollution Processes and Environmental Criteria (Ministry of Education), College of Environmental Science and Engineering, Nankai University, Tianjin 300350, China

\$ School of Public Health, North China University of Science and Technology, Tangshan 063000, China

${ }^{\S}$ College of Life Sciences, Nankai University, Tianjin 300071, China

*Correspondence: Qixing Zhou. E-mail: zhouqx@nankai.edu.cn. Tel: +86-22-23507800. Fax: +86-022-23501117.

The authors declare that they have no competing interests.

Number of pages: 11

Number of Figures: 8

Number of Tables: 2 


\section{Determination of $\mathbf{P b}$}

To evaluate the released $\mathrm{Pb}^{2+}$ from the 3 PbSe-NPs, $2000 \mu \mathrm{g} / \mathrm{ml} \mathrm{PbSe-NPs}$ (the concentration of the mixture which was intraperitoneally injected to rats) were suspended in saline and serum. After incubated at $37{ }^{\circ} \mathrm{C}$ for $12 \mathrm{~h}$, the mixture was centrifuged at $12000 \mathrm{rpm}$ for $30 \mathrm{~min}$. Then, the supernatants were collected and digested with $30 \% \mathrm{HNO}_{3}$, and the total $\mathrm{Pb}$ concentration was determined with the inductively coupled plasma-mass spectrometry (ICP-MS, PerkinElmer, ELAN DRC-e).

The $0.1 \mathrm{~g}$ testes or $1.0 \mathrm{ml}$ serum samples were digested with $4.0 \mathrm{ml}$ concentrated $\mathrm{HNO}_{3}$ and $1.0 \mathrm{ml} \mathrm{H} \mathrm{O}_{2}$ in a microwave digestion system (Shanghai Yiyao WX-4000, China). The digestion liquids were heated to dry and then $10 \mathrm{ml} 1 \% \mathrm{HCl}$ was added. The concentration of $\mathrm{Pb}$ in testes or serum was determined with the ICP-MS and expressed as $\mu \mathrm{g} / \mathrm{g}$ wet weight or $\mu \mathrm{g} / \mathrm{ml}$.

\section{Determination of Sperm Characteristics}

To evaluate the concentration of sperm, $5 \mu$ aliquot of epididymal sperm was diluted with $95 \mu \mathrm{l}$ saline, and $10 \mu \mathrm{l}$ of this diluted specimen was transferred into each of the counting chambers of the hemocytometer, then the concentration of sperm was manually counted under a light microscope at $400 \times$ magnification.

To evaluate the motility of sperm, $10 \mu \mathrm{L}$ DMEM solution containing sperm was placed on a microscope slide and a cover slip was placed over the droplet. 200 sperms were manually assessed at $400 \times$ magnification using a phase contrast microscope. The motility of sperm was evaluated within 2-4 min of their isolation from cauda epididymides. Sperm motility $=$ the amount of motile sperm in 200 sperms $/ 200 \times$ $100 \%$.

To evaluate sperm abnormality, the sperm were smeared on glass slides and allowed to dry in air. Then slides were stained with 1\% eosin-Y / 5\% nigrosin and 400 sperms were manually examined at $400 \times$ for morphological abnormalities. Sperm abnormality $=$ the amount of abnormal sperm in 400 sperms $/ 400 \times 100 \%$. 


\section{Western Blotting Analyses}

The protein concentrations were determined by the BCA method (Beyotime Institute of Biotechnology, Jiangsu, China). Proteins were loaded and separated by $12 \%$ sodium dodecyl sulfate-polyacrylamide gel electrophoresis (SDS-PAGE) for $100 \mathrm{~min}$ in an electrophoresis chamber and blotted to polyvinylidene fluoride (PVDF) membranes (Merck Millipore, IPVH00010) via a semi-dry electrophoretic transfer method. Then the membranes were blocked in 5\% powdered skimmed milk for $1.5 \mathrm{~h}$ at room temperature. After being washed three times with $1 \times \mathrm{TBST}$, membranes were incubated to primary antibodies against GRP78 (Cell Signaling Technology, CST, \#3183, 1:1000), Cleaved Caspase-3 (CST, \#9661, 1:1000), Caspase-12 (Abcam, ab18766, 1:200), Cyt c (Santa Cruz Biotechnology, sc-7159, 1:1000), Bax (CST, \#2772, 1:1000), Bcl-2 (CST, \#2870, 1:1000), and $\beta$-actin (CST, \#3700, 1:5000) at $4{ }^{\circ} \mathrm{C}$ overnight. After washed with $1 \times \mathrm{TBST}$ for 3 times, membranes were subsequently incubated with horseradish peroxidase-conjugated secondary antibodies (HRP-labeled Goat Anti-Rabbit IgG (H+L), Beyotime, A0239, 1:500; HRP-labeled Goat Anti-Mouse IgG (H+L), A0216, 1:1000) at room temperature for $2 \mathrm{~h}$. Protein bands were visualized by adding enhanced chemiluminescence reagent and analyzed with a gel documentation system (Gel Doc1000 and Multi-Analyst version 1.1, Bio-Rad, Hercules, CA, USA).

\section{Fertility Evaluations}

Female rats were examined each morning and the observations of the vaginal plug or vaginal smear were considered evidence of successful copulation. Once insemination was confirmed, the female and male rats were separated and housed individually in polycarbonate cages and the female rats were checked at least 3 times daily on days 19-21 of pregnancy to determine the time of delivery. The females were allowed to deliver spontaneously and nurse their pups until postnatal day 4 (PND 4). Total litter size and the numbers of living and dead pups were recorded and examined on PND 4.

The copulation success rate $(\%)=$ no. of female rats with successful copulation / no. of all female rats of each group $\times 100$. The fertility rate $(\%)=$ no. of pregnant 
female rats $/$ no. of female rats with successful copulation $\times 100$. The gestation rate $(\%)=$ no. of female rats which delivered live fetal rats / no. of pregnant female rats $\times$ 100. The fetus no. $=$ no. of fetal rats / no. of female rats which delivered live pups. The live fetuses rate $(\mathrm{PND} 4, \%)=$ no. of live fetus rats on postnatal day $4 /$ no. of fetal rats $\times 100$.

\section{Supplementary Figures}

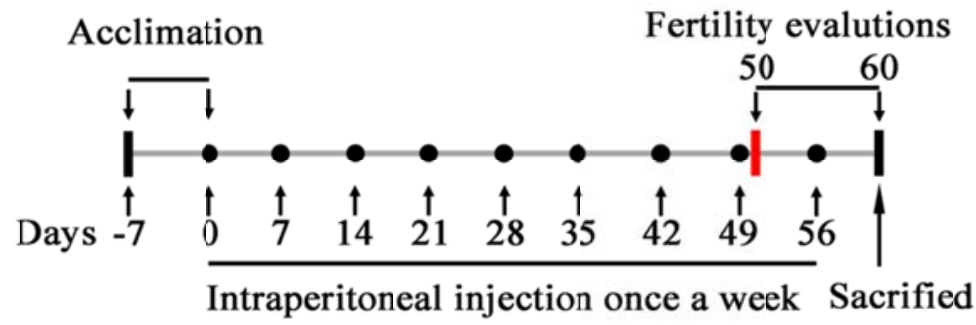

Figure S1. Scheme of repeatedly administration with PbSe-NPs. 
a
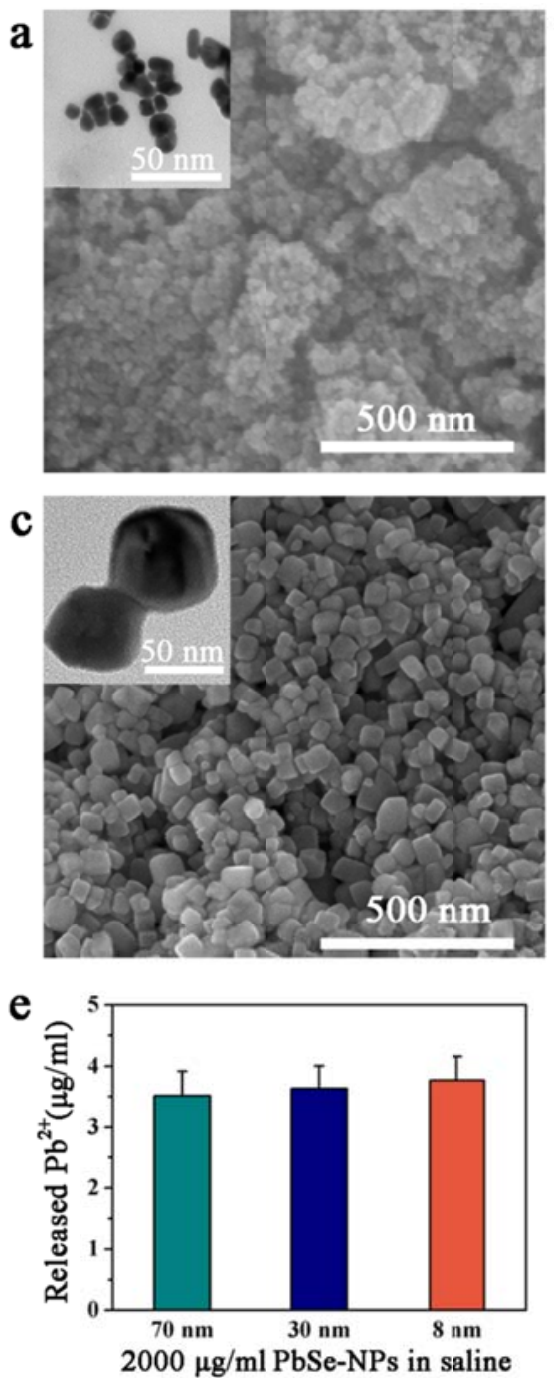
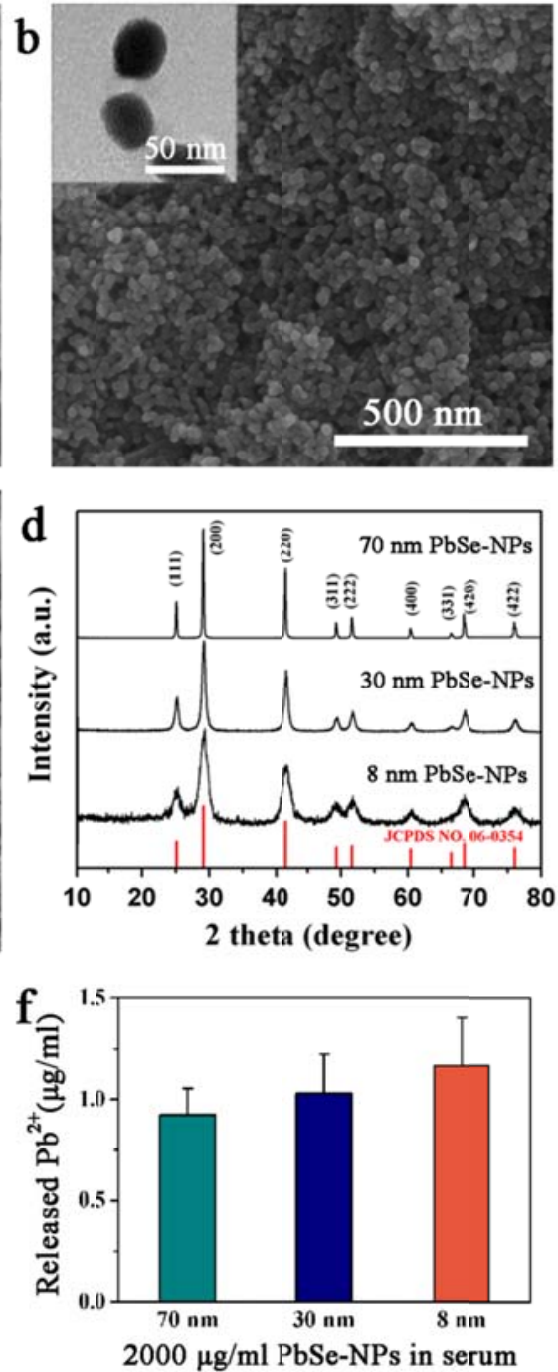

Figure S2. The characterization of the 3 PbSe-NPs. The SEM observation of $8 \mathrm{~nm}(\mathrm{a})$, $30 \mathrm{~nm}$ (b) and $70 \mathrm{~nm}$ (c) PbSe-NPs, the illustration in a, b and c is the representative TEM image, respectively; and X-ray diffraction (XRD) patterns (d) of the 3 PbSe-NPs. 

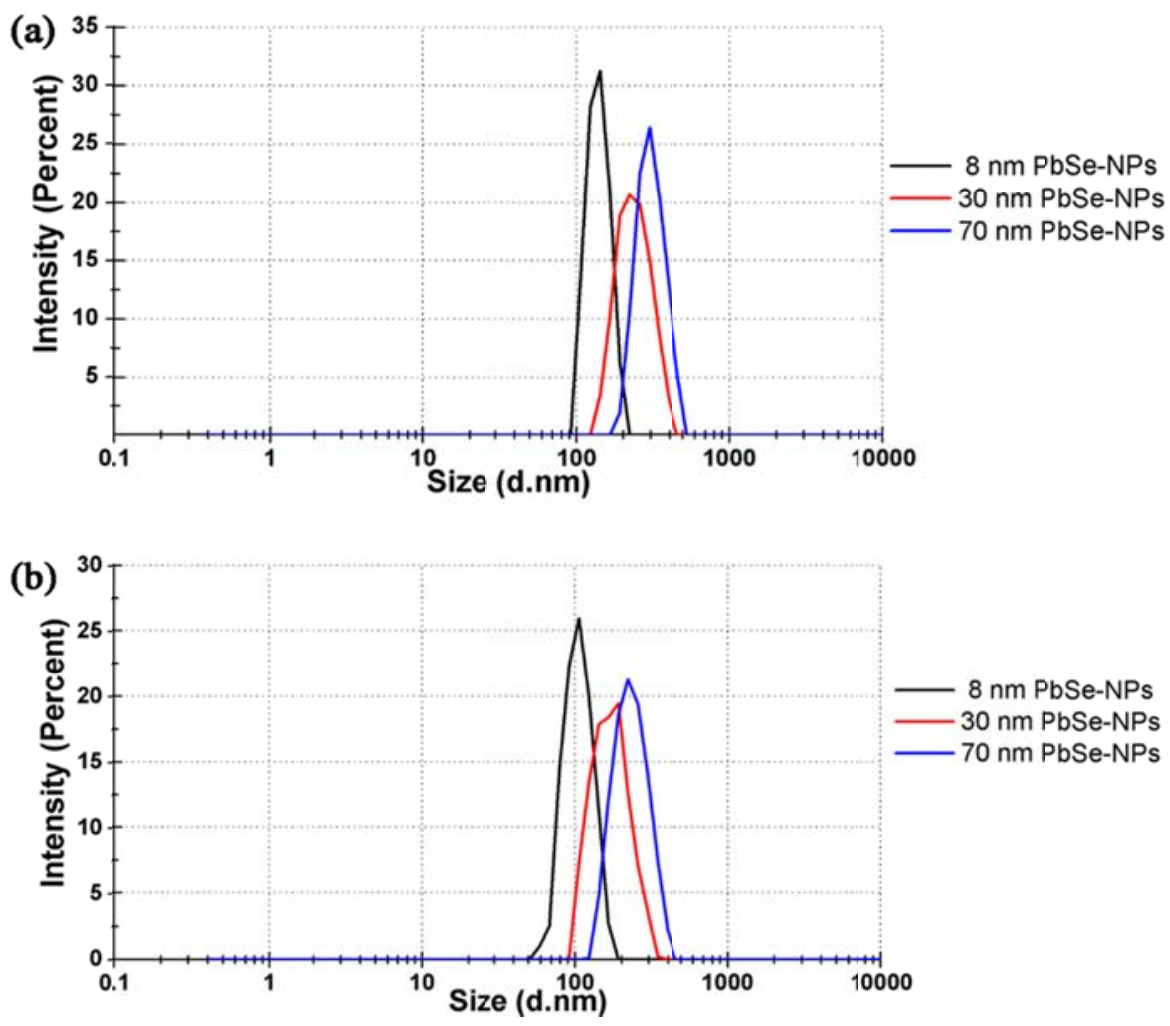

Figure S3. The hydrodynamic diameter $(\mathrm{pH}=7.0)$ of the 3 size PbSe-NPs. (a)

PbSe-NPs in saline; (b) PbSe-NPs in serum. 
(a)

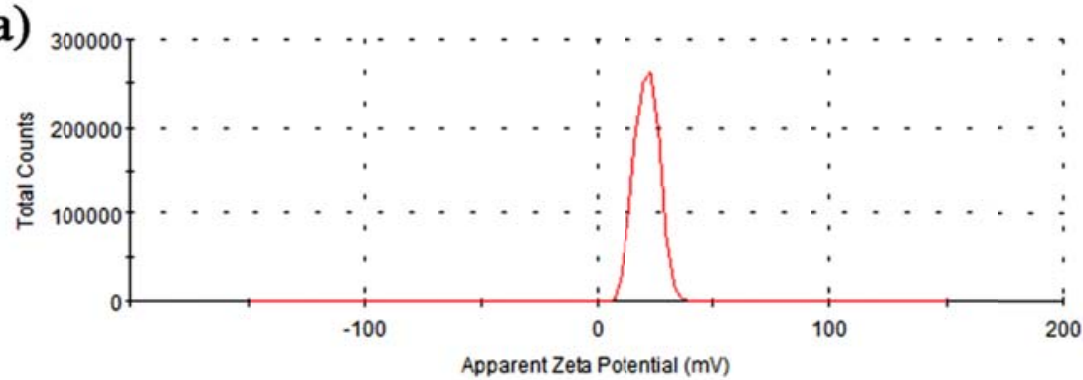

(b)

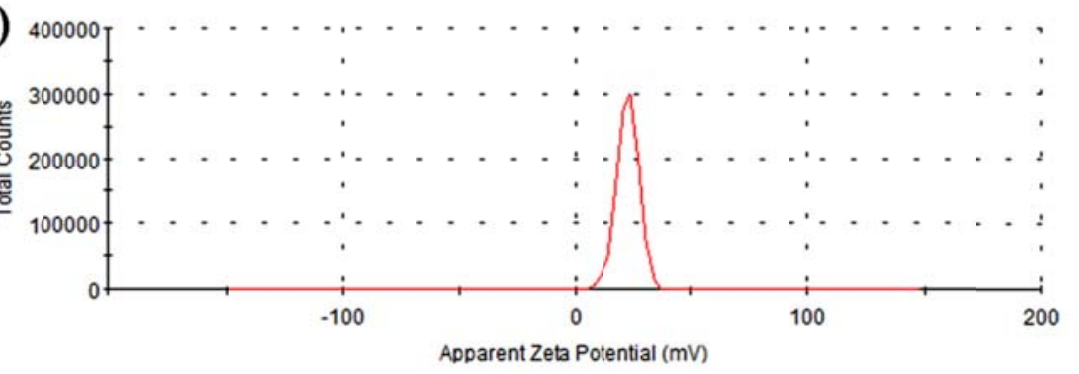

(c)

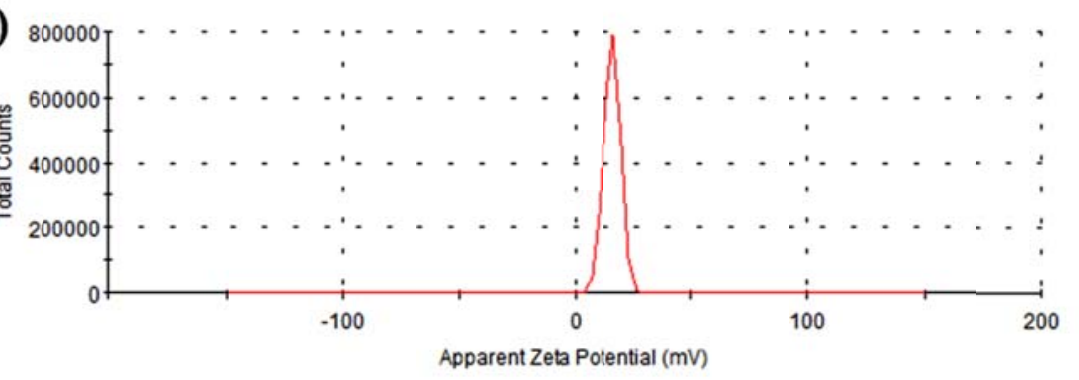

Figure S4. The zeta potential ( $\mathrm{pH}=7.0$ ) of the 3 PbSe-NPs: (a) $70 \mathrm{~nm}$ PbSe-NPs; (b) 30 nm PbSe-NPs; and (c) 8 nm PbSe-NPs.

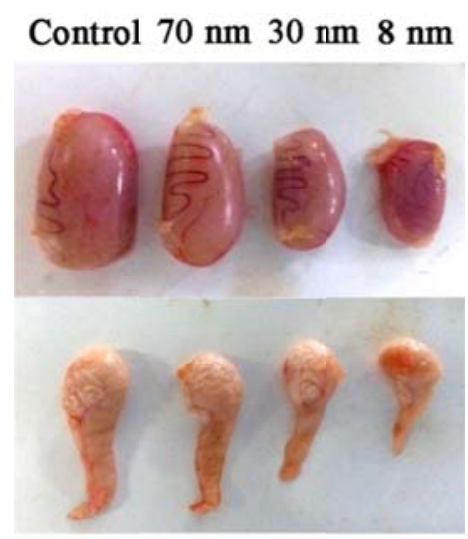

Figure S5. The representative diagrams of separated testes and epididymides in the control group and the $3 \mathrm{PbSe}-\mathrm{NPs}$ groups. 


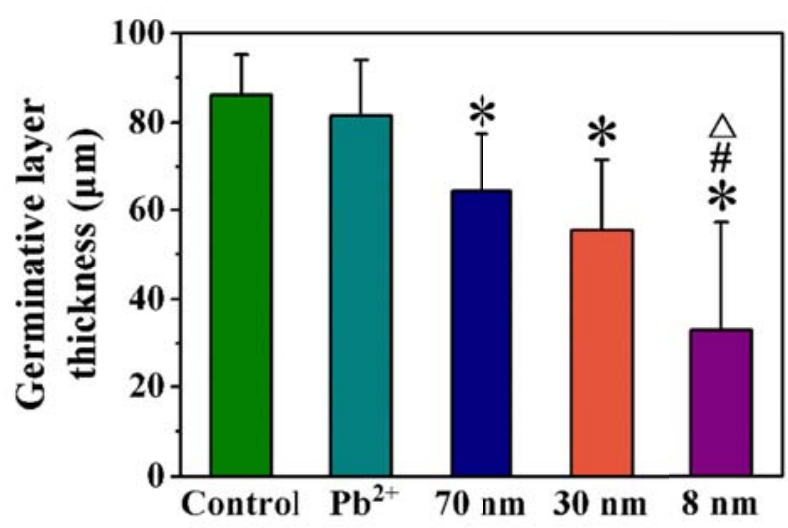

Figure S6. The thickness of the germinative layer in testes in all the groups. Data are shown as mean $\pm \mathrm{SD},{ }^{*} \mathrm{P}<0.05$ compared with the control group, ${ }^{\#} \mathrm{P}<0.05$ compared with $70 \mathrm{~nm}$ PbSe-NPs group, and ${ }^{\Delta} P<0.05$ compared with $30 \mathrm{~nm}$ PbSe-NPs group.

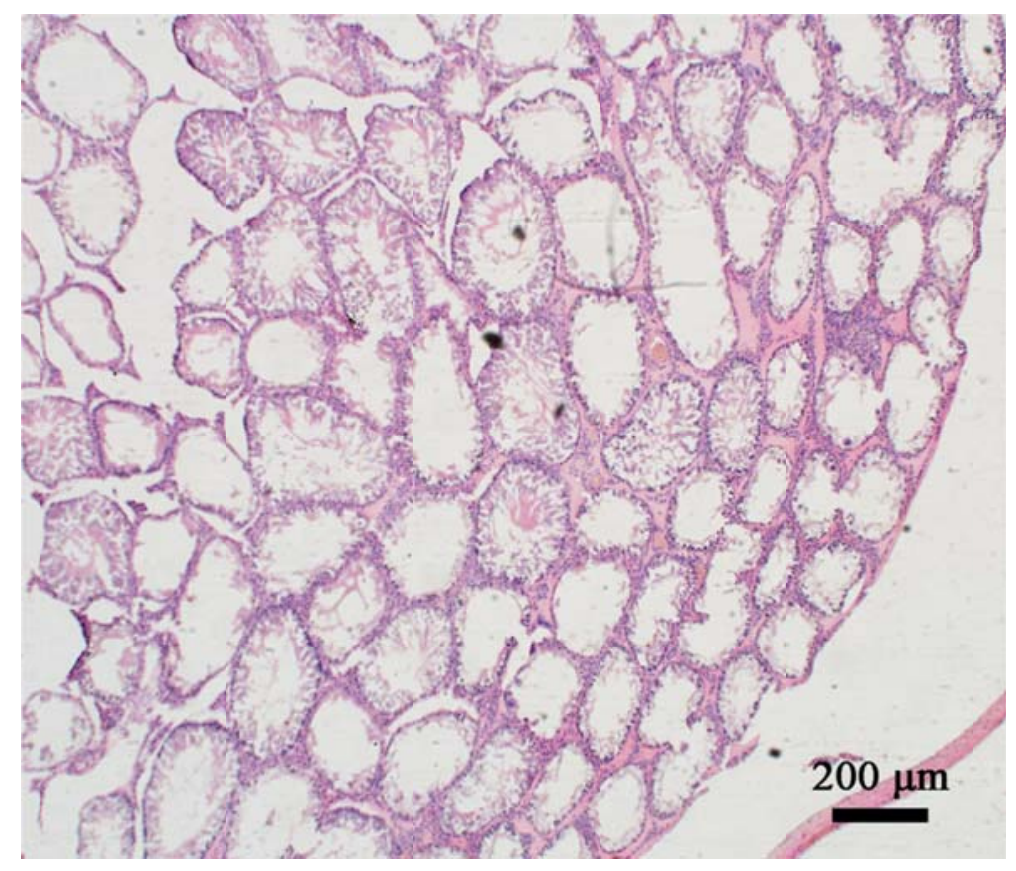

Figure S7. The respective pathologic color graph of seminiferous tubules in the $8 \mathrm{~nm}$ PbSe-NPs group was observed with the low magnification. 

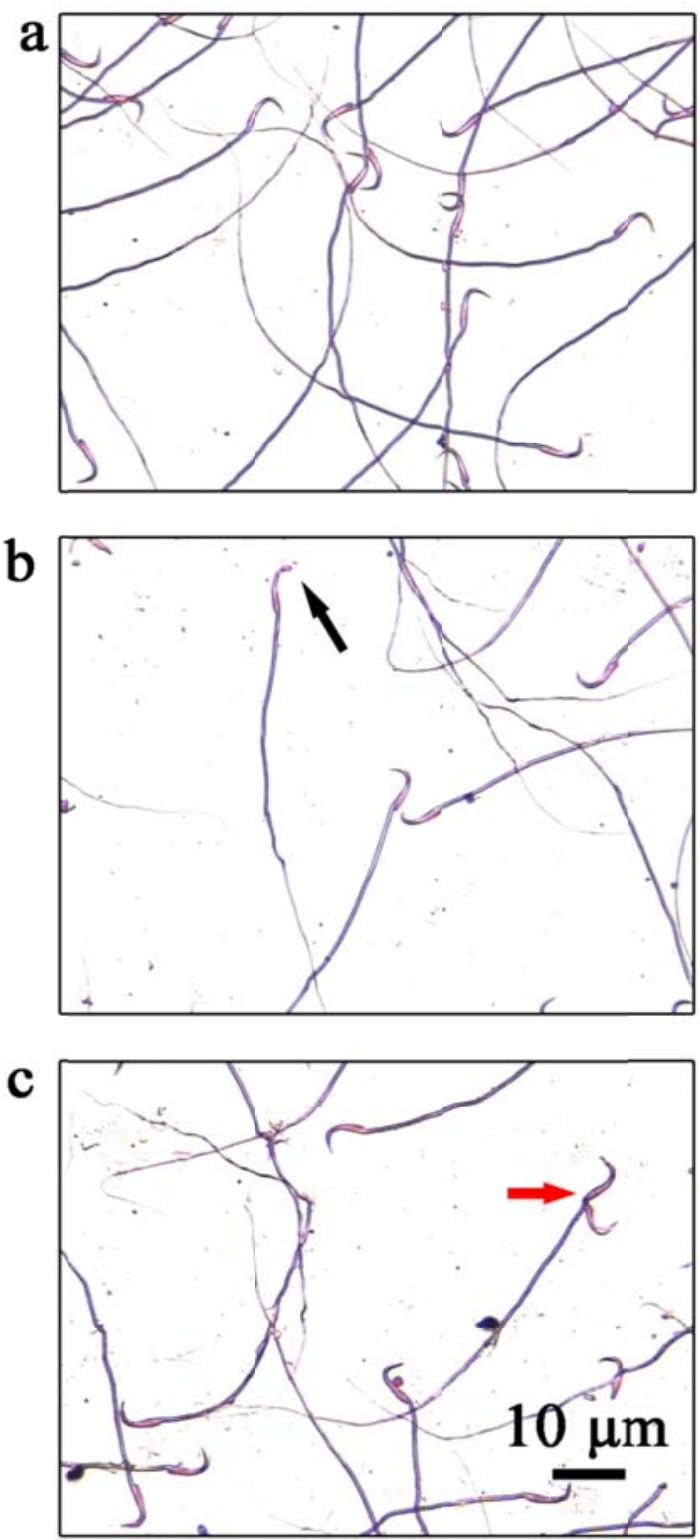

Figure S8. The respective images of sperms stained with $1 \%$ eosin- $\mathrm{Y} / 5 \%$ nigrosin: (a) sperms from the control group with the normal structure, morphological abnormalities such as hook less (black arrow in b) and bicephalic (red arrow in c) sperms were observed in the 8nm PbSe-NPs group (b and c). 

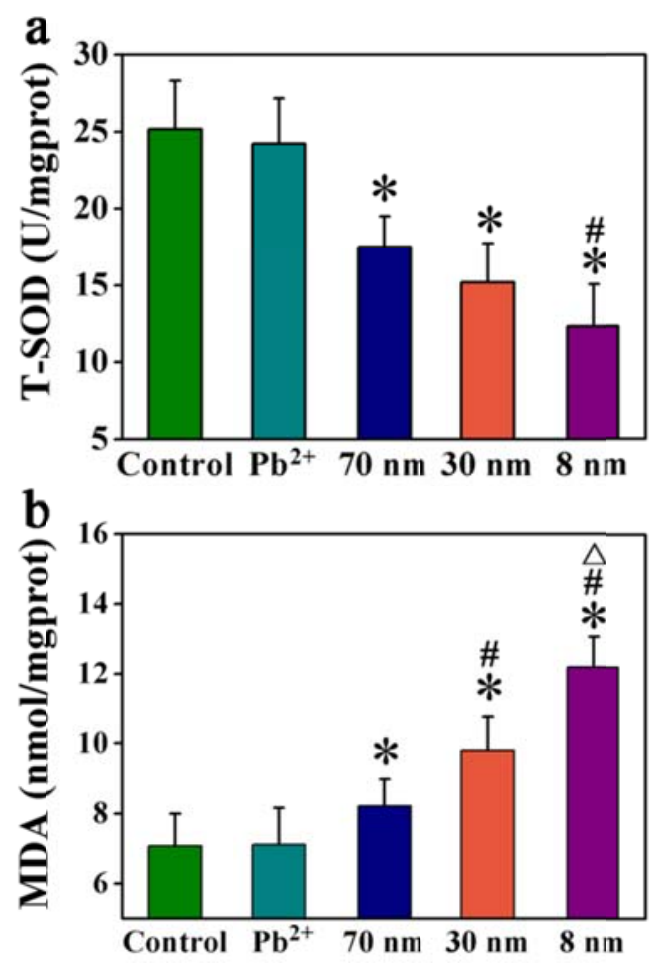

Figure S9. T-SOD (a) and MDA (b) levels in testes of a rat exposed to PbSe-NPs. Data are shown as mean $\pm \mathrm{SD}, n=8$ of each group. ${ }^{*} P<0.05$ compared with the control group, ${ }^{\#} \mathrm{P}<0.05$ compared with $70 \mathrm{~nm} \mathrm{PbSe}-\mathrm{NPs}$ group, and ${ }^{\Delta} \mathrm{P}<0.05$ compared with $30 \mathrm{~nm}$ PbSe-NPs group. 


\section{Supplementary Tables}

Table S1. The particle size, hydrodynamic diameter (DLS, $\mathrm{pH}=7.0$ ) and zeta potential $(\mathrm{pH}=7.0)$ of the $3 \mathrm{PbSe}-\mathrm{NPs}$.

\begin{tabular}{ccccc}
\hline PbSe-NPs & TEM (nm) & DLS (saline, nm) & DLS (serum, nm) & Zeta potential (mV) \\
\hline $70 \mathrm{~nm}$ & $70 \pm 29.6$ & $353 \pm 43.9$ & $241 \pm 58.8$ & $21.7 \pm 5.16$ \\
$30 \mathrm{~nm}$ & $30 \pm 11.2$ & $238 \pm 41.6$ & $170 \pm 47.37$ & $23.0 \pm 4.78$ \\
$8 \mathrm{~nm}$ & $8 \pm 3.4$ & $169 \pm 22.93$ & $106 \pm 28.97$ & $16.1 \pm 3.69$ \\
\hline
\end{tabular}

Table S2. Effects of PbSe-NPs on the fertility of rats.

\begin{tabular}{|c|c|c|c|c|c|}
\hline Group & $\begin{array}{c}\text { Copulation success } \\
\text { rate }(\%)\end{array}$ & $\begin{array}{c}\text { Fertility rate } \\
\text { (\%) }\end{array}$ & $\begin{array}{c}\text { Gestation rate } \\
(\%)\end{array}$ & Fetus no. & $\begin{array}{c}\text { Live pups rate } \\
\text { (PND4, \%) }\end{array}$ \\
\hline Control & $100(16 / 16)$ & $100(16 / 16)$ & $100(16 / 16)$ & $9.5(152 / 16)$ & $96.7(147 / 152)$ \\
\hline $\mathrm{Pb}^{2+}$ & $100(16 / 16)$ & $100(16 / 16)$ & $100(16 / 16)$ & $9.3(149 / 16)$ & $94.6(141 / 149)$ \\
\hline $70 \mathrm{~nm}$ & $100(16 / 16)$ & $100(16 / 16)$ & $100(16 / 16)$ & $8.8(141 / 16)$ & $91.5(129 / 141)$ \\
\hline $30 \mathrm{~nm}$ & $75(12 / 16)$ & $75(9 / 12)$ & $100(9 / 9)$ & $7.3(66 / 9)^{* \#}$ & $77.3(51 / 66)^{* \#}$ \\
\hline $8 \mathrm{~nm}$ & $62.5(10 / 16)$ & $60(6 / 10)$ & $100(6 / 6)$ & $5.3(32 / 6)^{* \# \Delta}$ & $62.5(20 / 32)^{* \# \Delta}$ \\
\hline
\end{tabular}

One male rat was paired with two female rats; each group has 8 male rats and 16 female rats. ${ }^{*} P<0.05$ compared with the control group, ${ }^{\#} P<0.05$ compared with 70 nm PbSe-NPs group, and ${ }^{\Delta} \mathrm{P}<0.05$ compared with $30 \mathrm{~nm}$ PbSe-NPs group. 